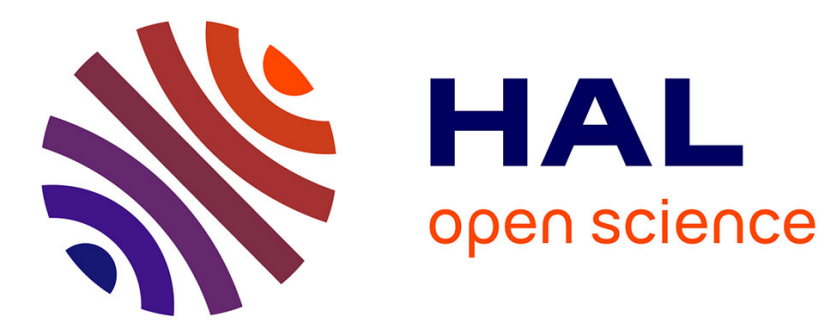

\title{
Molecular Dynamics Studies of Initiation in Energetic Materials
}

P. Haskins

\section{To cite this version:}

P. Haskins. Molecular Dynamics Studies of Initiation in Energetic Materials. Journal de Physique IV Proceedings, 1995, 05 (C4), pp.C4-595-C4-597. 10.1051/jp4:1995448 . jpa-00253754

\section{HAL Id: jpa-00253754 https://hal.science/jpa-00253754}

Submitted on 1 Jan 1995

HAL is a multi-disciplinary open access archive for the deposit and dissemination of scientific research documents, whether they are published or not. The documents may come from teaching and research institutions in France or abroad, or from public or private research centers.
L'archive ouverte pluridisciplinaire HAL, est destinée au dépôt et à la diffusion de documents scientifiques de niveau recherche, publiés ou non, émanant des établissements d'enseignement et de recherche français ou étrangers, des laboratoires publics ou privés. 


\title{
Molecular Dynamics Studies of Initiation in Energetic Materials
}

\author{
P.J. Haskins \\ Defence Research Agency, Fort Halstead, Sevenoaks, Kent, U.K.
}

\begin{abstract}
In this paper an overview of Molecular Dynamics simulations of chemically reacting systems is described. In particular, molecular dynamics simulations of shock initiation in a model energetic material are reported. The use of Molecular Dynamics to model thermal initiation and determine reactions rates in energetic materials is also discussed. Finally, the future potential of MD techniques for energetic materials applications is considered.
\end{abstract}

\section{INTRODUCTION}

Molecular Dynamics (MD) is the term used to describe the solution of the classical equations of motion (Newton's equations) for a set of molecules. A typical simulation is composed of hundreds, even thousands of atoms. Molecular Dynamics has been used successfully to study the properties of liquids, small rigid molecules, and even large molecules such as proteins. However, it is only very recently that this technique has been applied to the study of energetic materials[1,2,3,4]. A major break-through has been the development of complex inter-atomic potentials such as the Abell-Tersoff potential, which allow chemical reactions to occur through incorporation of a many-body term. This has allowed the study of model reactive systems. Indeed, MD studies offer the prospect of a greatly improved understanding of the coupling between the shock physics and the chemistry of energetic materials.

\section{RESULTS AND DISCUSSION}

Molecular Dynamics studies at Fort Halstead have involved development of the in-house code DYNAMITE, incorporating a many-body empirical potential, and subsequent use of the code to study detonation phenomena. In particular, the initiation, growth and steady state detonation characteristics have been quantified for a model system. The influence of exothermicity, activation barriers, and inital shock conditions have all been addressed. The work reported in this paper concerns both thermal and shock initiation in a homogeneous system of diatomic molecules. Both 2D and 3D versions of the code have been employed which incorporate the empirical Abell-Tersoff potential. This potential is basically a pair potential and is the sum of a repulsive term, a bonding term, and a non-bonding term. The many-body characteristics of the potential arise through the bonding and non-bonding terms being dependent on third body interactions. 
The MD simulations were set up as either regular 2D or 3D (alpha FCC) lattices of heteronuclear diatomic molecules at zero pressure. The atomic mass of nitrogen (14 amu) was used for the atoms in the model. Two sets of binding energies were used in a series of simulations. In one set of simulations a binding energy of $5 \mathrm{eV}$ was used for the products $\mathrm{A}-\mathrm{A}$ and $\mathrm{B}-\mathrm{B}$ while $2 \mathrm{eV}$ was used for the unreacted material. In a further set of simulations, $4 \mathrm{eV}$ was used for the products and $1 \mathrm{eV}$ for the reactant. The MD code DYNAMITE was written in Fortran 77 and includes periodic boundary conditions, in all dimensions for the thermal initiation studies, but not on the x-axis (shock direction) for shock studies. The code can be run on the DRA Cray or Silicon Graphics computers. Typical run times are a few hours on the Cray YMP for simulations of about 10ps and a few thousand atoms.

The particular aims of the work reported here were to demonstrate that the model system gives chemically reasonable thermal reactions kinetics, and a shock initiation response curve of the correct form for a homogeneous explosive. Considering the thermal initiation studies first, both $2 \mathrm{D}$ and 3D simulations were carried out on a square/cubic box of molecules at various inital temperatures. This model was used to study the reaction processes occurring, the times to reaction, and to obtain the specific heats $(\mathrm{Cv})$. The specific heats $(\mathrm{Cv})$ showed a slight increase with increasing temperature. At the highest temperatures $\left(\mathrm{ca} .1500^{\circ} \mathrm{K}\right.$ ) which could be studied (before reaction) the model gave: $\mathrm{Cv}=$ $1.28 \mathrm{R}(0.18 \mathrm{cal} / \mathrm{g})$ for the $2 \mathrm{D}$ model, and $\mathrm{Cv}=1.69 \mathrm{R}(0.24 \mathrm{cal} / \mathrm{g})$ for the $3 \mathrm{D}$ version. A wide variety of reactions have been observed. The most common are:

$$
2 \mathrm{AB}--\rightarrow \mathrm{A}_{2}+\mathrm{B}_{2}, \quad 2 \mathrm{AB}--->\mathrm{A}_{2}+2 \mathrm{~B}, \quad \mathrm{~B}+\mathrm{AB} \rightarrow->\mathrm{B}_{2}+\mathrm{A}
$$

Back reactions and concerted triatomic processes have also been observed. Reaction times have been shown to be very similar in both the $2 \mathrm{D}$ and $3 \mathrm{D}$ version of the model over a range of temperatures. One feature that is worth noting is that classic "induction time" behaviour is observed at the lower temperatures.

In shock initiation simulations, the initial shocks were introduced by impact of a flyer composed of the same molcules as the acceptor lattice. For the shock initiation studies we have examined the influence of the pulse duration, which is determined by the flyer plate thickness, and the effect of the activation barrier on the threshold pulse amplitudes (flyer velocities) required to achieve "detonation". From these studies it is possible to make some general comments on the properties of the model. Firstly, provided the initial stimulus is sufficient a steady-state chemically sustained shock (detonation) is achieved. This travels at ca. $6 \mathrm{~km} / \mathrm{s}$ with the parameters used, and is independent of the initial shock. Secondly, detonation results in almost complete conversion of the initial heteronuclear molecules to the more stable homonuclear species.

Initiation in homogeneous explosives is experimentally, observed to show a weak dependence on pulse duration except, for very short high-energy pulses, but to require a minimum particle velocity. For homogeneous systems, it has been widely demonstrated that initiation can be described by use of Thermal Explosion Theory. We have carried out MD calculations to see if our model system is consistent with this behaviour. The essential concept of Thermal Explosion Theory is that after the initial shock heating there is an induction time before significant chemical reaction. Provided the pulse duration is long enough to ensure that no rarefaction cools the material before the induction period is complete, then initiation is assumed to occur. It should be noted that the theory is not strictly valid for very strong shocks since some reaction occurs without an induction period and the hydrodynamics and the chemistry cannot be de-coupled. However, it is still very instructive to base an approximate criterion on the time required to obtain a significant degree (eg. $90 \%$ ) of reaction. For the 2D simulations five thicknesses of flyer plates ranging between 5 and $37.5 \mathrm{~A}^{0}$ were employed. In addition two A-B well depths were considered namely, $2 \mathrm{eV}$ and $1 \mathrm{eV}$. For each potential the particle velocity shock velocity relationship (Hugoniot) was determined. Threshold initiation velocities were ascertained for each flyer thickness and the Hugoniot used to plot the results as threshold energies versus pulse duration. The results of this study showed that the threshold curves had the correct form with a strong dependence on pulse duration for short pulses and weak dependence for longer pulses. Reasonable fits to the MD results were obtained with a simple thermal explosion model based on a single-step exothermic Arrhenius reaction. The threshold condition for the thermal explosion model was taken to be $90 \%$ reaction. The Arrhenius parameters needed to provide the fits were shown to be chemically sensible, with the activation energies amounting to about $75 \%$ of the unimolecular bond strengths. The model with an A-B well depth of $1 \mathrm{eV}$ was fitted with an activation energy of half that of the model with a $2 \mathrm{eV}$ well depth, and the same pre-exponential factor. The simulations were repeated with the $3 \mathrm{D}$ code using a similar arrangement of molecules. A total of six flyer thicknesses were examined, but only one potential (with an $\mathrm{A}-\mathrm{B}$ well depth of $2 \mathrm{eV}$ ) was employed. The results from the $3 \mathrm{D}$ simulations were 
plotted as threshold energies against pulse duration as for the $2 \mathrm{D}$ results. As might be expected, very similar results were obtained to the 2D calculations. Despite the Hugoniot and specific heat being significantly different from the 2D model (as expected), the Arrenhius parameters needed to provide a thermal explosion fit were very similar.

In general, the results obtained with both the $2 \mathrm{D}$ and $3 \mathrm{D}$ versions of the model gave good agreement with thermal explosion theory. This is despite the fact that the predictions made with thermal explosion theory were strictly outside it's range of validity. Furthermore, an arbitary $90 \%$ reaction criterion and single step decomposition scheme were used. Since it is known that there are a number of key reactions this is clearly a gross simplification, and a two step model would be expected to give a much improved fit. It should be noted though, that the agreement between the classical thermal explosion model and the MD results strongly suggests that the MD model supports a classical thermal explosion model for shock initiation. Comparison with 'real' systems can be made by extrapolating the thermal explosion fit to a $2 \mathrm{~mm}$ thick flyer plate. When this is done a threshold velocity of $2.1 \mathrm{~km} / \mathrm{s}$ is obtained (for the potential with an A-B well depth of $2 \mathrm{eV}$ ). This is not an unrealistic value for a real homogeneous explosive. As would be expected for a homogeneous system the Walker-Wasley critical energy $\left(\mathrm{P}^{2} \mathrm{t}\right)$ criterion does not provide a good fit to the initiation threshold curve. It is interesting to note however, that it does give an approximate fit to the shorter duration pulse results.

\section{FUTURE WORK}

Looking towards the future, it is hoped that MD studies might be able to address heterogeneous explosives where hotspots are important. For these types of explosives it would be interesting to extend MD studies to lattices with defects. However, the problem of treating realistic hot spot dimensions (ca. $10^{-7}-10^{-5} \mathrm{~m}$ ) needs to be resolved before this is achieveable. It should be noted that some apparently heterogeous explosives, for example TATB, appear to have a significant homogeneous behaviour.

Future work will investigate the possibility of including more realistic ab-initio potentials. Ab-initio potentials could be obtained by inclusion of either a Molecular Orbital (MO) or Density Functional (DF) code within the MD code. This could then be used to generate ab-initio potentials directly for all short range interactions and an empirical potential could be used for long-range forces. However, the problems of incorporating either an MO or DF code directly into a MD code are non-trivial. It is hoped that the thermal explosion model might be extended to incorporate a coupled Arrhenius kinetic scheme which could be compared to the MD results. Finally, the ultimate aim of the work is to employ a kinetic model, derived from the MD work, directly into a hydrocode in order to simulate longer time (microsecond) and larger scale behaviour.

\section{REFERENCES}

[1] P J Haskins and M D Cook, "Molecular Dynamics Studies of Shock Initiation in a model Energetic Material" in Proceedings of the APS Topical Conference on Shock Compression of Condensed Matter, North Holland, 1994, pp 1341-1344.

[2] D W Brenner, M L Elert and C T White, "Incorporation of Reactive Dynamics in Simulations of Chemically-Sustained Shock Waves" in Proceedings of the APS Topical Conference on Shock Compression of Condensed Matter, North Holland, 1989, pp 263-266.

[3] D W Brenner, "Molecular Potentials for Simulationg Shock-Induced Chemistry" in Proceedings of the APS Topical Conference on Shock Compression of Condensed Matter, North Holland, 1991, pp 115-121.

[4] D H Robertson, D W Brenner, M L Elert and C T White "Simulations of Chemically-Sustained Shock Fronts in a Model Energetic Material" in Proceedings of the APS Topical Conference on Shock Compression of Condensed Matter, North Holland, 1991, pp 123-126 